\title{
Gender-Related Changes in Magnetocardiographically Determined Fetal Cardiac Time Intervals in Intrauterine Growth Retardation
}

\author{
PETER VAN LEEUWEN, SVEN SCHIERMEIER, SILKE LANGE, ANITA KLEIN, DANIEL GEUE, WOLFGANG HATZMANN, \\ AND DIETRICH H.W. GRÖNEMEYER
}

\begin{abstract}
Department of Biomagnetism [P.L, S.L., A.K., D.G.], Department of Radiology and Microtherapy [D.H.W.G.], Grönemeyer Institute of Microtherapy, University Witten/Herdecke, 44799 Bochum, Germany; Department of Obstetrics [S.S., W.H.], Marienhospital, University of Witten/Herdecke, 58452 Witten, Germany
\end{abstract}

\begin{abstract}
Prenatal growth deficiencies as well as gender have been associated with cardiovascular disease in later life. It is also known that the duration of fetal cardiac time intervals (CTI) are dependent on fetal development. The aim of this work was to examine the relationship between fetal CTI in healthy and intrauterine growth retardation (IUGR) fetuses, taking gender into account. A total of 269 magnetocardiograms (MCG) were obtained in 47 healthy and 27 IUGR pregnancies. In each signal-averaged MCG, durations of CTI were determined. Age- and heart rate-corrected values were compared between normal and IUGR fetuses separately with respect to gender. Overall, there was an association between atrial and ventricular conduction times and estimated fetal body weight. In female fetuses, IUGR was associated with shorter P WAVE, PQ segment, PR interval, and QRS complex and longer STT and QT intervals. For males, this was so only for $\mathrm{P}$ wave, QRS complex, and STT interval. The shortening of conduction times in IUGR may be explained by reduced cardiac muscle mass associated with lower body weight. On the other hand, the gender-specific differences, particularly in the IUGR fetuses may be due to hormonal factors.
\end{abstract}

(Pediatr Res 59: 820-824, 2006)

$\mathrm{O}^{\prime}$ n the basis of both electrocardiographic and magnetocardiographic measurements, the durations of fetal cardiac time intervals have been successfully assessed in the course of pregnancy (1-4). Most studies have described a prolongation of atrial and ventricular depolarization times with advancing gestational age, and this has generally been attributed to overall growth and the concomitant increase in fetal cardiac muscle mass $(4,5)$, based on the following rationale. The augmentation in the mass and dimensions of the fetal heart during the second and third trimester has been quantified on the basis of ultrasound recordings (6,7). Furthermore, it has been shown that the weight of the fetal heart is not only dependent on gestational age but also on fetal body weight (8). Overall, in mammals, a relationship between body mass and cardiac conduction times has been described (9). Thus, an indirect relationship between depolarization times and body

Received November 16, 2005; accepted February 4, 2006.

Correspondence: Peter Van Leeuwen, Ph.D., Department of Biomagnetism, Grönemeyer Institute of Microtherapy, Universitätsstr. 142, 44799 Bochum, Federal Republic of Germany; e-mail: petervl@microtherapy.de

DOI: 10.1203/01.pdr.0000219300.95218.bb weight can be assumed: higher body weight is indicative of greater cardiac muscle mass as well as larger cardiac dimensions resulting in greater distances that a depolarization wave must travel. Accordingly, one may expect that fetal conduction times will to some degree reflect the growth status of the fetus.

Perinatal physiologic differences due to gender may be seen as a case in point. It is known that male neonates tend to have higher birth weights than females (10). It thus seems consistent that, in infancy, male QRS complex duration has been found to be longer than that of female (11). Prenatally, gender-based differences in intraventricular conduction times have been suspected (1), and recently we have been able to confirm significantly longer QRS duration in male fetuses from about the 30th week of gestation onward (5). This concurs with gender-based differences in fetal weight that become more predominant as term approaches (12). Significant correlations between gender, birth weight, and conduction times have also been confirmed by ECG traces obtained during labor (13).

Taken together, this background would suggest that, in IUGR, specific fetal CTI may be shortened and that this shortening may be modulated by the gender of the fetus. As prenatal growth deficiencies have been associated with cardiovascular pathologies in later life (14) and gender also plays a role in the expression of cardiovascular disease (15), understanding gender-specific prenatal cardiac function should be of interest. The aim of this study was to determine CTI in IUGR fetuses on the basis of magnetocardiography and to compare the results in males and females to those found in normally developed fetuses.

\section{METHODS}

Subjects. The subjects in this study included 74 women. Of these, 47 had normal pregnancies (28 male, 19 female fetuses). The IUGR group consisted of 27 cases ( 9 male, 18 female fetuses). Normal pregnancies excluded cases

Abbreviations: CTI, cardiac time intervals; IUGR, intrauterine growth retardation; MCG, magnetocardiography; pGH, placental growth hormone 
of fetal growth retardation, congenital heart disease or arrhythmia, and maternal hypertension, diabetes, or nicotine abuse. IUGR pregnancies were diagnosed on the basis of ultrasound examination (estimated weight $<10$ th percentile with respect to gestational age) and the growth retardation was confirmed postpartum by a pediatrician. Excluded from the IUGR group were pregnancies with fetal arrhythmia or congenital heart disease. The women in normal pregnancy were slightly older (age: $32.2 \pm 3.4$ versus $27.3 \pm 4.3$ y), had been pregnant more often (gravida: $2.1 \pm 1.2$ versus $1.6 \pm 0.9$ ) and had born more children (parity: $0.7 \pm 0.6$ versus $0.4 \pm 0.6$ ) than those with IUGR fetuses (values expressed as mean $\pm \mathrm{SD}$ ).

In the normal pregnancies, we recorded and evaluated 230 fetal magnetocardiograms (FMCG) between the 15th and 42nd week of gestation, 116 (50.4\%) in male fetuses. In the 27 IUGR fetuses, 39 FMCG recordings were obtained between the 27th and 39th week of gestation of which $15(38.5 \%)$ were in male fetuses. Table 1 compares the postnatal status of the healthy and the IUGR fetuses. The study was approved by the local ethics committee and all mothers gave written informed consent.

Data acquisition. FMCG were acquired using either a 61-channel biomagnetometer (Magnes 1300C, 4D Neuroimaging, San Diego, CA) or a 37channel system (Krenikon, Siemens, Erlangen, Germany), which have been described in detail elsewhere (5). We have previously been able to show that the use of these two systems in the calculation of fetal CTI lead to comparable results and no bias is to be expected (16). For data acquisition, the mothers were in a supine position and the biomagnetometer was placed as close to the abdomen as possible while avoiding skin contact. The 61-channel system was positioned symmetrically over the abdomen, the lower rim over the pubis, and the upper rim approximately over the xiphoid. The smaller 37-channel system was positioned such that channels at the center of the sensor displayed high-amplitude fetal QRS complexes. Data were recorded for $5 \mathrm{~min}$ at a sampling rate of $1 \mathrm{kHz}$ and with a bandpass of $1-200 \mathrm{~Hz}$. To reduce the effects of external noise, all measurements were performed in a standard shielded room (AK3b, Vacuumschmelze, Hanau, Germany).

Data analysis. In each set of data, the signal components of the maternal heart beats were identified and digitally subtracted from the signal traces (16). Subsequently, fetal beats were identified on the basis of a representative fetal QRS signal template, which was constructed from 20-30 manually selected fetal QRS traces of similar morphology. Fetal PQRST courses to be averaged (including generally $\gg 300$ beats) were chosen on the basis of a good correlation $(r \geq 0.90)$ to the template. This approach ensured that signal segments distorted by artifacts, movement, etc., did not corrupt the averaged data and delivered a characteristic signal trace (16). In the averaged data, the onsets and endpoints of the $\mathrm{P}$ wave, QRS complex, and T wave were defined according to published standards (17), and independently determined (and corrected when necessary) by two experienced investigators. We determined the consecutive, nonoverlapping (disjunct) intervals as follows: P wave, PQ segment, QRS complex, ST segment, and T wave. Furthermore, the following, commonly used composite CTI were examined: PR interval (= P wave $+\mathrm{PQ}$ segment) and QT interval (= QRS complex + ST segment + T wave). As the QT interval is determined by age-related changes in QRS duration as well as by changes in momentary heart rate, we also determined the STT interval (= ST segment $+\mathrm{T}$ wave) to be able to examine the QT component primarily susceptible to rate changes. In some traces, signal quality made definitive identification of the timing of an event difficult, particularly in the assessment of the $\mathrm{T}$ wave (16). If the reasonable identification of the timing was not possible, the corresponding CTI were not determined. For details see Refs. 5 and 16.

Statistics. Values are generally expressed as mean $\pm \mathrm{SD}$. To examine the relationship between the CTI and fetal weight, the latter was estimated for each FMCG acquisition in the IUGR fetuses on the basis of ultrasound examinations (based on the Hadlock formula), which were performed within approximately $48 \mathrm{~h}(1.4 \pm 2.1 \mathrm{~d})$ of almost all FMCG acquisitions. This data were not available for the healthy fetuses who were recruited independent of clinical surveillance. Thus, for these fetuses, we estimated body weight at the time of the last available FMCG recording using the actual birth weight and values from published standard birth weight data over a range of gestational ages (12). For each fetus, this was done by subtracting the difference between standard birth weight at the time of birth and the actual birth weight from the standard birth weight at the time of the last MCG recording. Spearman's $r$ was used to quantify correlation between weight and the CTI.

The duration of the determined CTI were compared between the normal and IUGR fetuses separately with respect to gender. As specific CTI may be related to both gestational age and RR interval (i.e., heart rate), these dependencies were estimated on the basis of multiple regression, whereby linear and nonlinear models were examined. For this estimation, the data of the healthy fetuses in an age range comparable to that of the IUGR fetuses ( $\geq 27$ wk gestation) were used. For all regressions, the dependencies due to repeated measurements were considered by performing a robust regression using a modified Huber/White/Sandwich estimator of variance (18). For any interval, the model that demonstrated the best fit, based on the coefficient of determination, was chosen as most appropriate. On the basis of the best fitting model, the CTI of the normal and IUGR fetuses were corrected, arbitrarily to the 35th week of gestation and to an RR interval of $420 \mathrm{~ms}$. As the above approach takes the effect of heart rate into account, we avoided using the often-applied but disputed Bazett correction (19). The corrected CTI values of the normal and IUGR fetuses were compared using the Mann-Whitney $U$ test and statistical significance was assumed at a level of 0.05 .

\section{RESULTS}

The heart rates of the IUGR and normal groups were not significantly different over a comparable gestational age: normal versus IUGR males, $143.5 \pm 9.1$ and $141.6 \pm 8.2 \mathrm{bpm}$; normal versus IUGR females, $143.3 \pm 8.8$ and $144.0 \pm 8.5$ bpm (see also Fig. 1). On the other hand, comparison of the CTI plotted with respect to gestational age suggested differences between the healthy and the IUGR fetuses for various intervals. Overall, the depolarization times ( $\mathrm{P}$ wave, PR interval, QRS complex) tended to be shorter and ventricular repolarization times (STT and QT interval) slightly longer in the IUGR group (Fig. 1). Also, gender differences were apparent. In the male fetuses, the QRS complex values in IUGR lay clearly at and below the lower values of the healthy fetuses whereas the female IUGR QRS values showed a larger overlap in the range of the values of the healthy fetuses. For the $\mathrm{P}$ wave, the values of the female IUGR fetuses tended to differ more from those of the healthy when compared with the differences in the male fetuses. To a degree this was also true for the PQ segment and the PR interval. In the STT interval (and consequently, QT interval), the values of the male IUGR fetuses varied less (range, $56 \mathrm{~ms}$ ) and lay in the upper half of the normal values whereas the female dispersed over a wider range $(98 \mathrm{~ms})$. With respect to fetal weight, a positive correlation between the CTI and the estimated weight was found in particular for the depolarization times (Table 2), irregardless of group.

The investigation of the relationship between the CTI and gestational age and/or RR interval showed that, on the whole, the type of dependency was similar for males and females

Table 1. Fetal outcome characteristics

\begin{tabular}{|c|c|c|c|c|c|c|c|c|}
\hline & No. & $\begin{array}{c}\text { Gestational } \\
\text { age at birth } \\
\text { (wk) }\end{array}$ & $\begin{array}{c}\text { Birth weight } \\
(\mathrm{g})\end{array}$ & $\begin{array}{c}\text { Crown heel length } \\
(\mathrm{cm})\end{array}$ & $\begin{array}{c}\text { Head } \\
\text { circumference } \\
(\mathrm{cm})\end{array}$ & $\begin{array}{c}\text { Apgar score } \\
5 \mathrm{~min} \\
\end{array}$ & $\begin{array}{c}\text { Apgar score } \\
10 \mathrm{~min} \\
\end{array}$ & $\mathrm{pH}$ \\
\hline IUGR all & 27 & $37.0 \pm 3.0 *$ & $2198 \pm 550 *$ & $44.8 \pm 4.3^{*}$ & $31.8 \pm 2.4^{*}$ & $9.3 \pm 1.0$ & $9.6 \pm 0.7$ & $7.3 \pm 0.1$ \\
\hline IUGR male & 9 & $38.4 \pm 1.7$ & $2502 \pm 229$ & $46.8 \pm 2.0$ & $32.6 \pm 1.5$ & $9.0 \pm 1.3$ & $9.4 \pm 0.9$ & $7.3 \pm 0.1$ \\
\hline IUGR female & 18 & $36.3 \pm 3.3$ & $2046 \pm 604 \dagger$ & $43.8 \pm 4.9$ & $31.4 \pm 2.8$ & $9.4 \pm 0.7$ & $9.7 \pm 0.6$ & $7.3 \pm 0.1$ \\
\hline
\end{tabular}

$* p<.0005$ normal vs IUGR; $\dagger p<0.05$ IUGR male $v s$ IUGR female (Mann-Whitney $U$ test). 

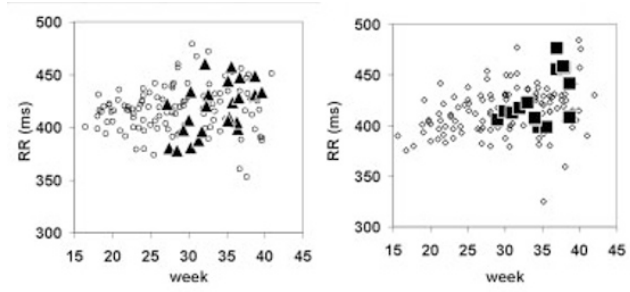

Figure 1. Duration of the RR interval and cardiac time intervals with respect to gestational age, separately for female healthy $(\bigcirc)$ or growth-retarded $(\mathbf{\Delta})$ and male healthy $(\diamond)$ or growth-retarded $(\boldsymbol{\square})$ fetuses.
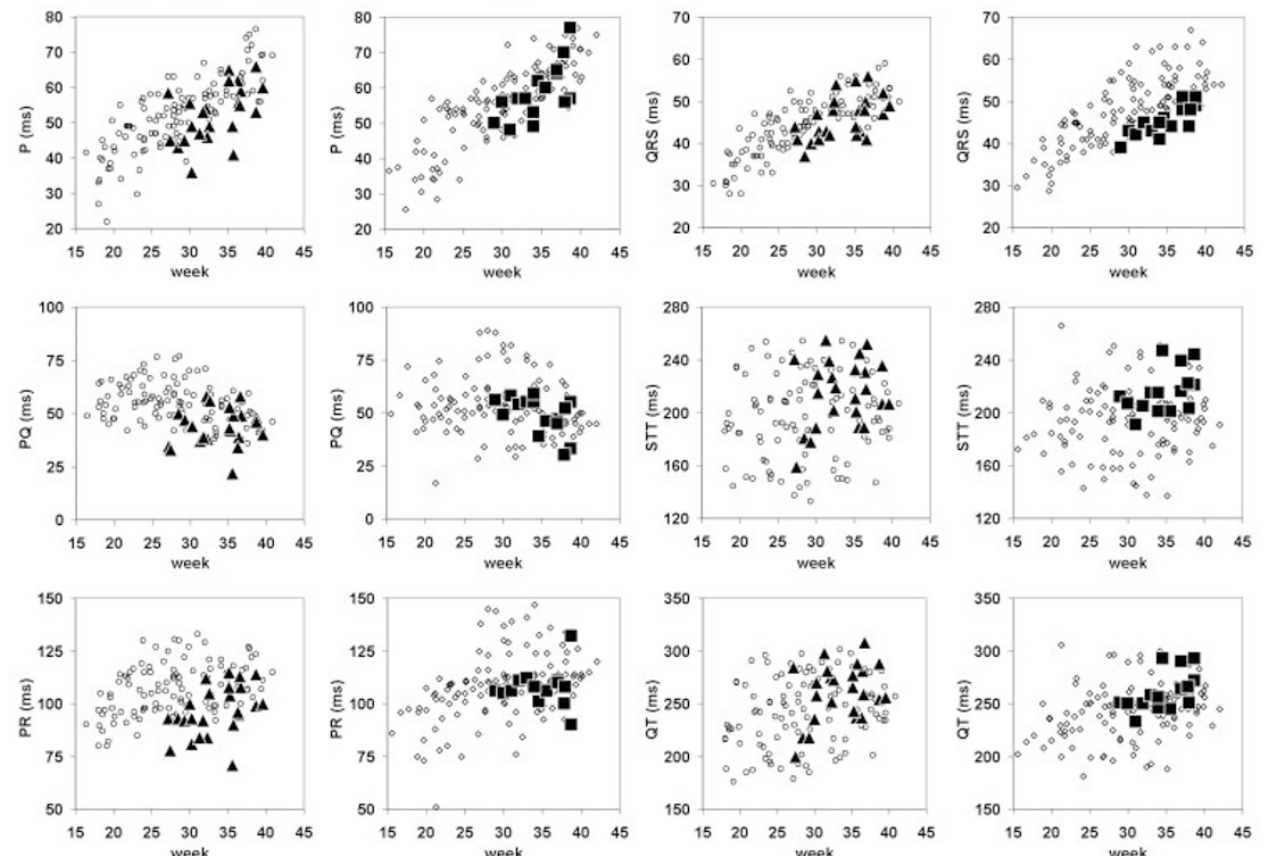

Table 2. Correlations between the CTI and body weight (Spearman $r$ and corresponding $p$ values)

\begin{tabular}{|c|c|c|c|c|c|c|c|c|c|c|}
\hline & \multicolumn{2}{|c|}{$P$} & \multicolumn{2}{|c|}{$\mathrm{PQ}$} & \multicolumn{2}{|c|}{ QRS } & \multicolumn{2}{|c|}{ ST } & \multicolumn{2}{|c|}{$\mathrm{T}$} \\
\hline IUGR (ultrasound) & 0.64 & 0.000 & -0.07 & 0.698 & 0.47 & 0.004 & -0.10 & 0.560 & 0.30 & 0.084 \\
\hline
\end{tabular}

Fetal weight was estimated a) in the IUGR fetuses on the basis of ultrasound at each FMCG acquisition and b) for the healthy fetuses (N) at the time of the last prepartum FMCG on the basis of standard birth weight values (see text).

Table 3. Results of the multiple regression in the data of the healthy males and females showing the dependency of each fetal cardiac time interval on gestational age and/or RR interval

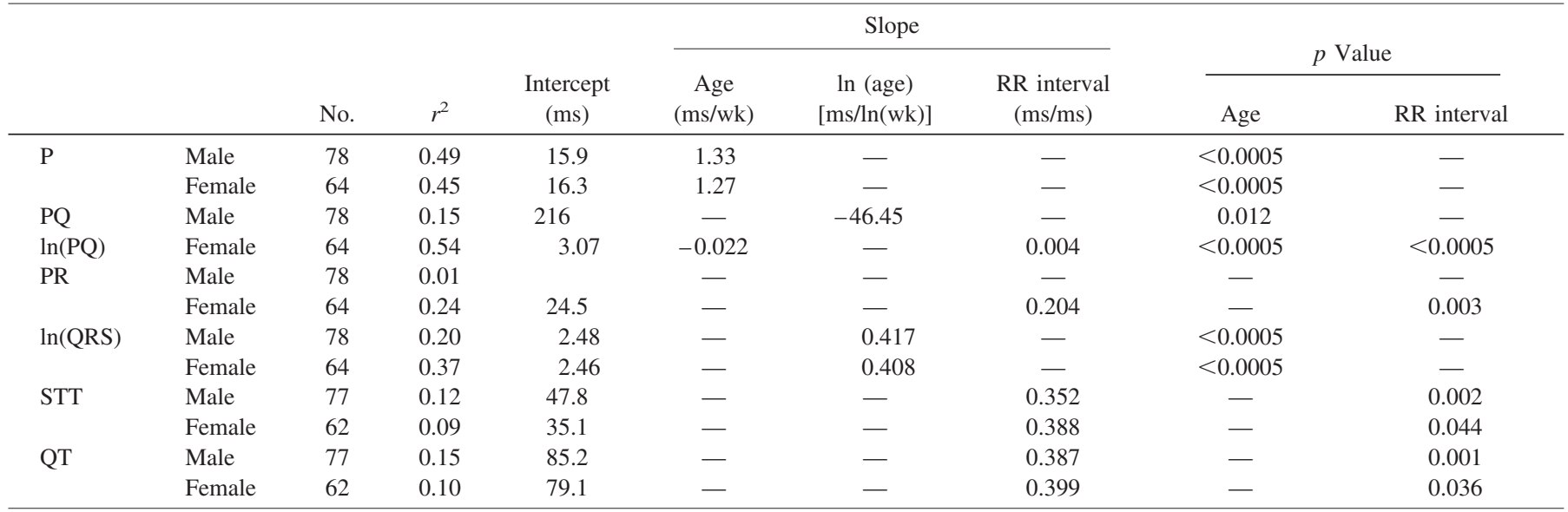

$r^{2}$, coefficient of determination; ln, natural logarithm. 
(Table 3). The $\mathrm{P}$ wave and QRS complex were dependent on age; STT and QT interval were dependent on RR interval. The exception was the PQ segment, with the females showing a dependency on both age and RR interval, whereas, in the males, only age played a role. This also affected the results for the PR interval. The fits were slightly better for the male data with respect to the P wave and the STT and QT intervals; the fits for the female data were substantially better with respect to the PQ segment, PR interval, and QRS complex.

The age- and heart rate-corrected results confirmed the visual impression gained from Figure 1. The depolarization times were significantly shorter in the IUGR fetuses, whereby the difference in the males was more marked in the QRS times and in the females more in the P wave duration (Fig. 2). The PQ segment was shortened only in the female IUGR fetuses, leading to a significantly more rapid overall atrioventricular conduction solely for this gender group. Ventricular repolarization was significantly lengthened in both IUGR groups, slightly more clearly in the males. Due to the opposing
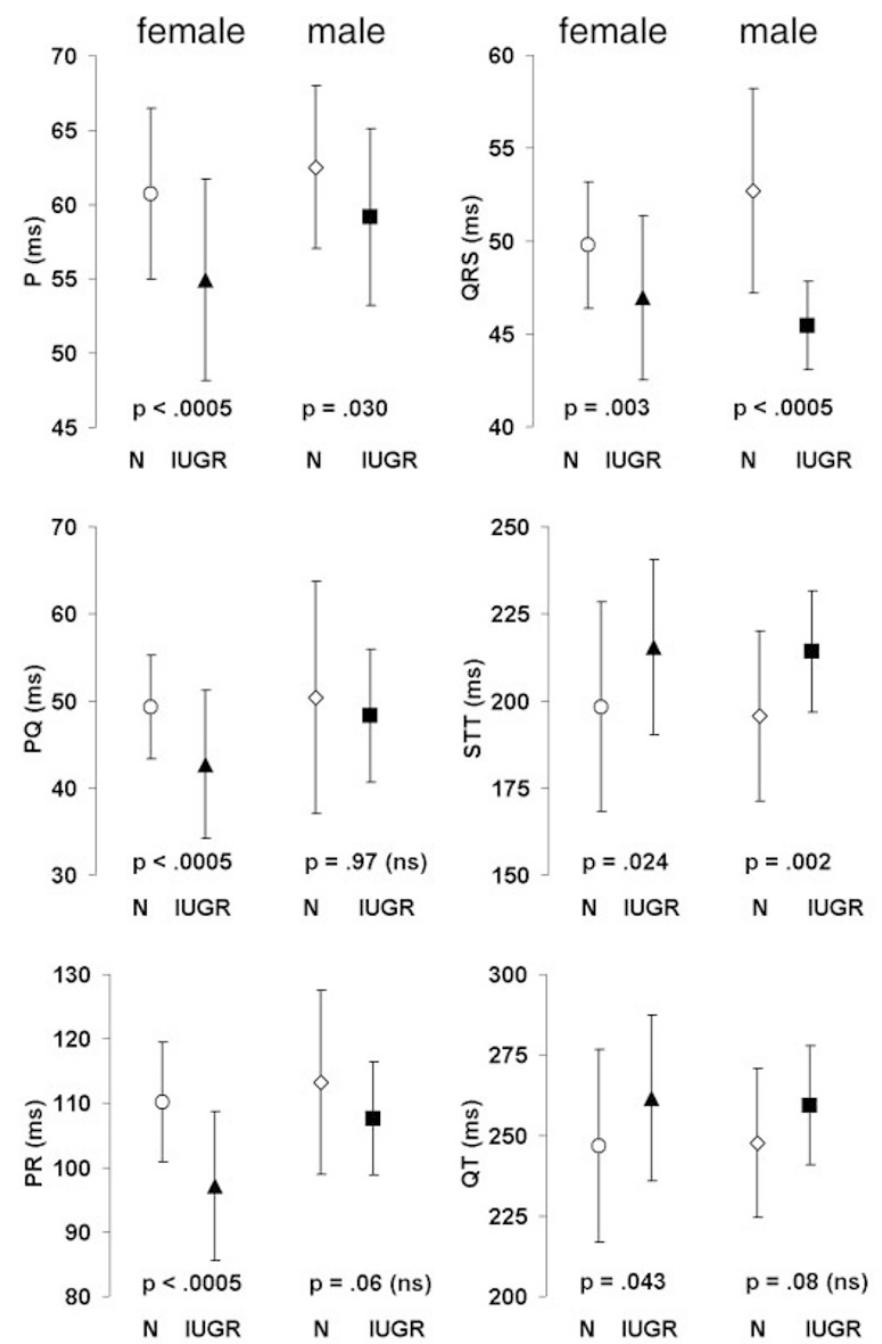

Figure 2. Mean $( \pm \mathrm{SD})$ duration of the fetal cardiac time intervals in female healthy $(\bigcirc)$ or growth-retarded $(\boldsymbol{\Delta})$ and male healthy $(\diamond)$ or growth-retarded $(\boldsymbol{\square})$ fetuses. Values were age and rate corrected (35th week of gestation and $420 \mathrm{~ms}$ RR interval), $p$ values for the comparison between healthy $(N)$ and growth-retarded (IUGR) fetuses. NS, not significant. directions of change in the QRS complex and STT interval, the differences in QT times between normal and IUGR fetuses were only minimal.

\section{DISCUSSION}

Overall, the results presented in this study confirm the relationship between the duration of CTI and fetal weight. However, the results suggest that there are subtle differences in the CTI of male and female fetuses. The longer QRS times in male fetuses corroborates gender-based data acquired from the pediatric, child, and adult ECG $(11,20,21)$. As QRS duration has been shown to be longer in heavier fetuses and children $(1,20)$ and males generally weigh more than females, the gender difference in ventricular conduction time likely reflects the discrepancy in body size and weight. Our data analysis has further shown that, in normal pregnancies, there are gender differences in the dependencies of the CTI on factors such as age and heart rate, in particular during atrioventricular activation. Taking these dependencies into consideration, we were able to show not only that the CTI of the growth-retarded fetuses were altered compared with those of the healthy fetuses but also that these changes were not the same between males and females (Fig. 2). Thus, although all IUGR fetuses demonstrated a tendency for shorter depolarization times, this was most clearly seen in the females during atrial activity, while for the males the difference was more obvious during ventricular depolarization. Whereas the overall shortening in the IUGR fetuses may be presumed to result from the lower cardiac muscle mass associated with reduced fetal weight, the gender-specific differences in CTI shortening require further elucidation.

In this respect, it is interesting to note that in the adult, gender differences in activation time are more multifaceted. Not only shorter female QRS duration but also longer PR intervals, ventricular activation times, and longer repolarization times have been reported in young adult females compared with their male counterparts (22). Differentiated examination of the atrioventricular activation time has shown that the time between the onset of His bundle activity and the onset of ventricular depolarization is shorter in women (23), and it was speculated that hormonal factors may play a role in this. The endocrine mechanisms involved in intrauterine growth are not well understood, but it is known that hormonal regulation differs between the sexes during gestation (24-26). To the degree that steroid compounds influence differences in conduction times in the fetus, they may help explain the differing effect that growth retardation has in male and female fetuses with respect to these times. For example, pGH is thought to play a role in fetal growth. It has been shown that maternal serum pGH concentrations are lower in women with low weight fetuses (27) and also that male pregnancies tend to have lower serum pGH levels (26). Taken together with the fact that overall fetal growth is associated with heart growth, this may help explain the more clearly shortened male QRS durations in the growth-retarded group.

On the other hand, the more pronounced shortening of atrial depolarization times in the female IUGR fetuses may result 
from gender differences in estradiol concentrations. Animal studies have shown that elevated estradiol levels may prolong atrial effective refractory periods (28) and differences in estradiol concentrations between the genders in infants have been observed (29). It is thus conceivable that prenatal differences in such hormone levels in association with gender and growth retardation may lead to the observed differences in atrial conduction observed here.

Although the duration of repolarization (STT interval) of the growth-retarded fetuses lay in the range of the values of the healthy fetuses, there was a tendency toward longer times in the IUGR group. This delay may be due to repolarization inhomogeneities resulting from the compromised state of the fetuses. Indeed, epidemiologic studies have associated low birth weight with heart disease (14) and results from animal studies suggest that growth retardation may be associated with gender-specific cardiac remodeling (30). Thus, cardiac repolarization disturbances may also have been present in the IUGR fetuses. Furthermore, gender differences may also be reflected in the tendency of lower Apgar values in the male IUGR fetuses, suggesting that ischemia may have been more likely in this group. Our results must however be viewed with caution as the fetal $\mathrm{T}$ wave, due to its low-amplitude, lowfrequency character (16), is more often than not difficult to discern and the low reliability in $\mathrm{T}_{\text {end }}$ determination will confound the interpretation of the results.

Overall, all the CTI of growth-retarded females were significantly altered with respect to those of the healthy female fetuses. For the males, this was true only for the P wave, QRS complex, and STT interval. This difference may reflect gender-specific reactions to growth retardation. On the other hand, this may simply be due to the fact that the IUGR females, on average, were born more prematurely and had lower birth weights, crown-rump lengths, and head circumference than the IUGR males. The latter cannot, however, account for the tendency for gender-specific shortening of the atrial and ventricular conduction times. This must be explained by some other mechanism, as suggested above. Taken together, the results indicate that the precise determination of fetal de- and repolarization times give new insights into normal and pathologic fetal development. The CTI most certainly reflect growth status and may also be related to hormonal changes in pregnancy.

\section{REFERENCES}

1. Brambati B, Pardi G 1980 The intraventricular conduction time of fetal heart in uncomplicated pregnancies. Br J Obstet Gynaecol 87:941-949

2. Taylor MJ, Smith MJ, Thomas M, Green AR, Cheng F, Oseku-Afful S, Wee LY, Fisk NM, Gardiner HM 2003 Non-invasive fetal electrocardiography in singleton and multiple pregnancies. BJOG 110:668-678

3. Quinn A, Weir A, Shahani U, Bain R, Maas P, Donaldson G 1994 Antenatal fetal magnetocardiography: a new method for fetal surveillance? Br J Obstet Gynaecol 101:866-870
4. Stinstra J, Golbach E, van Leeuwen P, Lange S, Menendez T, Moshage W, Schleussner E, Kaehler C, Horigome H, Shigemitsu S, Peters M 2002 Multicentre study on the fetal cardiac time intervals using magnetocardiography. BJOG 109:1235-1243

5. Van Leeuwen P, Lange S, Klein A, Geue D, Grönemeyer DH 2004 Dependency of magnetocardiographically determined fetal cardiac time intervals on gestational age, gender and postnatal biometrics in healthy pregnancies. BMC Pregnancy Childbirth 4:6

6. St. John Sutton MG, Gewitz MH, Shah B, Cohen A, Reichek N, Gabbe S, Huff DS 1984 Quantitative assessment of growth and function of the cardiac chambers in the normal human fetus: a prospective longitudinal echocardiographic study. Circulation 69:645-654

7. Firpo C, Hoffman JI, Silverman NH 2001 Evaluation of fetal heart dimensions from 12 weeks to term. Am J Cardiol 87:594-600

8. Guihard-Costa AM, Menez F, Delezoide AL 2002 Organ weights in human fetuses after formalin fixation. Pediatr Dev Pathol 5:559-578

9. Meijler FL 1985 Atrioventricular conduction versus heart size from mouse to whale. J Am Coll Cardiol 5:363-365

10. Kramer MS 1987 Determinants of low birth weight: methodological assessment and meta-analysis. Bull World Health Organ 65:663-737

11. Rijnbeek PR, Witsenburg M, Schrama E, Hess J, Kors JA 2001 New normal limits for the paediatric electrocardiogram. Eur Heart J 22:702-711

12. Alexander GR, Kogan MD, Himes JH 1999 1994-1996 U.S. singleton birth weight percentiles for gestational age by race, Hispanic origin, and gender. Matern Child Health J 3:225-231

13. Morgan M, Symonds EM 1991 Natural history determinants of the time constants of the fetal electrocardiogram. Eur J Obstet Gynecol Reprod Biol 42:S9-S13

14. Barker DJP, Gluckman PD, Godfrey KM, Harding JE, Owens JA, Robinson JS 1993 Fetal nutrition and cardiovascular disease in adult life. Lancet 341:938-941

15. Mendelsohn ME, Karas RH 2005 Molecular and cellular basis of cardiovascular gender differences. Science 308:1583-1587

16. Van Leeuwen P, Lange S, Klein A, Geue D, Zhang Y, Krause HJ, Grönemeyer D 2004 Reproducibility and reliability of fetal cardiac time intervals using magnetocardiography. Physiol Meas 25:539-552

17. Grimm B, Haueisen J, Huotilainen M, Lange S, Van Leeuwen P, Menendez T, Peters MJ, Schleussner E, Schneider U 2003 Recommended standards for fetal magnetocardiography. Pacing Clin Electrophysiol 26:2121-2126

18. Williams RL 2000 A note on robust variance estimation for cluster-correlated data. Biometrics 56:645-646

19. Franz MR 1994 Time for yet another QT correction algorithm? Bazett and beyond J Am Coll Cardiol 23:1554-1556

20. Vaksmann G, Elkohen M, Duhamel A, Godart F 1996 Signal-averaged electrocardiography in healthy children: influence of age, body size, and gender. PACE 19 (11 Pt 1):1629-1634.

21. Macfarlane PW, McLaughlin SC, Devine B, Yang TF 1994 Effects of age, sex, and race on ECG interval measurements. J Electrocardiol 27:14-9

22. Storstein L, Bjornstad H, Hals O, Meen HD 1991 Electrocardiographic findings according to sex in athletes and controls. Cardiology 79:227-236

23. Taneja T, Mahnert BW, Passman R, Goldberger J, Kadish A 2001 Effects of sex and age on electrocardiographic and cardiac electrophysiological properties in adults. Pacing Clin Electrophysiol 24:16-21

24. Kaplan SL, Grumbach MM The ontogenesis of human foetal hormones 19762. Luteinizing hormone (LH) and follicle stimulating hormone (FSH). Acta Endocrinol 81:808-829

25. Herruzo AJ, Mozas J, Alarcon JL, Lopez JM, Molina R, Molto L, Martos J 1993 Sex differences in serum hormone levels in umbilical vein blood. Int J Gynaecol Obstet 41:37-41

26. Chellakooty M, Skibsted L, Skouby SO, Andersson AM, Petersen JH, Main KM, Skakkebaek NE, Juul A 2002 Longitudinal study of serum placental GH in 455 normal pregnancies: correlation to gestational age, fetal gender, and weight. J Clin Endocrinol Metab 87:2734-2739

27. Chellakooty M, Vangsgaard K, Larsen T, Scheike T, Falck-Larsen J, Legarth J, Andersson AM, Main KM, Skakkebaek NE, Juul A 2004 A longitudinal study of intrauterine growth and the placental growth hormone $(\mathrm{GH})$-insulin-like growth factor I axis in maternal circulation: association between placental $\mathrm{GH}$ and fetal growth. J Clin Endocrinol Metab 89:384-391

28. Chen YJ, Lee SH, Hsieh MH, Hsiao CJ, Yu WC, Chiou CW, Chen SA 1999 Effects of 17 beta-estradiol on tachycardia-induced changes of atrial refractoriness and cisapride-induced ventricular arrhythmia. J Cardiovasc Electrophysiol 10:587-598

29. Schmidt IM, Chellakooty M, Haavisto AM, Boisen KA, Damgaard IN, Steendahl U, Toppari J, Skakkebaek NE, Main KM 2002 Gender difference in breast tissue size in infancy: correlation with serum estradiol. Pediatr Res 52:682-686

30. Battista MC, Calvo E, Chorvatova A, Comte B, Corbeil J, Brochu M 2005 Intra-uterine growth restriction and the programming of left ventricular remodelling in female rats. J Physiol 565:197-205 